\title{
DELAY IN DENTAL DEVELOPMENT AND VARIATIONS IN ROOT MORPHOLOGY ARE OUTCOMES OF THE COMPLEX ADAPTIVE SYSTEM ASSOCIATED WITH THE NUMERICAL VARIATION OF HYPODONTIA
}

\author{
LEO CHEN $^{1}$, HELEN LIVERSIDGE $^{1}, \mathrm{KE} \mathrm{CHEN}^{2}$, MAURO FARELLA $^{3}, \mathrm{SADAF}$ SASSANI ${ }^{4}$, \\ DILAN PATEL ${ }^{4}$, AZZA AL-ANI $^{4} \&$ ALAN BROOK ${ }^{4}$ \\ ${ }^{1}$ Queen Mary University, London. \\ ${ }^{2}$ University of Liverpool, England. \\ ${ }^{3}$ University of Otago, Otago, New Zealand. \\ 4) University of Adelaide, South Australia.
}

\begin{abstract}
The development of the dentition has the general characteristics of a complex adaptive system. Hypodontia is a developmental variation with not only a reduced number of teeth but also the teeth formed are smaller in size, have different crown and root morphology and are delayed in development. We have formed a multicentre, multidisciplinary collaborative study to investigate this complex system from its initiation to its outcome: from genotype with genetic/epigenetic/ environmental interactions to the accurate measurement of the phenotypic outcome. This paper reports an initial study of the root morphology and dental age component of the phenotype of the hypodontia patient compared to controls. The sample consists of orthodontic patients, 30 males and 30 females with hypodontia and 60 controls matched for age, gender and ethnicity. The material studied is the orthopantomographic radiograph of each patient. From these the number and site of each congenitally missing tooth is recorded. The number and shape of roots of each formed tooth are scored. Using the MATLAB computer programming platform, the distance between specific points on the crown and root, their area and hence the crown/ root ratio is computed, and the stages of dental development of each tooth scored; the degree of root development of the second permanent molar is particularly valuable in comparing between hypodontia patients and controls. By combining investigations from different stages of this biological complex adaptive system, we are using dental development, which is an accessible, non-invasive and accurately measurable paradigm to increase understanding of general development.
\end{abstract}

Keywords: Complex system, developments measures, hypodontia, radiographs.

\section{INTRODUCTION}

Developmental systems in biology may well behave as complex systems. It has been shown by Brook and O'Donnell [1] and Brook et al. [2] that dental development has the general characteristics of a complex adaptive system and that it is an accessible, non-invasive model for general development. The opportunity has arisen to examine the developmental process in relation to a specific variation of the number of teeth formed during development in some individuals. In hypodontia, together with the congenital absence of some teeth, those that are formed are smaller in size, have different crown and root morphology, and are delayed in development. We have formed a multicentre, multidisciplinary collaborative study to investigate this complex system from its initiation to its outcome: that is from the genotype with genetic/epigenetic/environmental interactions to the accurate measurement of the phenotypic outcome.

The aim of this part of the overall study is to investigate the root morphology and dental age component of the phenotype of the hypodontia patients compared to the controls. 


\section{MATERIALS, PATIENTS AND METHODS}

The sample consists of 120 orthodontic patients, 30 males and 30 females with hypodontia and 60 controls matched for age, gender and ethnicity. The age range of the patients was: hypodontia - males, 10.2 to 21.0 years; females, 10.8 to 19.3 years and controls - males, 10.1 to 17.3 years; females, 10.6 to 19.2 years. The material studied is the orthopantomographic radiograph of each patient. From these the number and site of each congenitally missing tooth in each patient is recorded.

The number and shape of the roots of each formed tooth is then scored. Using the MATLAB computer programming platform, we have developed programs to compute the distance between specific points on the crown and root, their area and hence the crown/root ratio is computed.

The stages of dental development of each tooth are also scored; the degree of root development of the second permanent molar is particularly valuable in comparing between these hypodontia patients and the controls.

\section{METHOD DEVELOPMENT/DISCUSSION}

The number of congenitally missing teeth is an indication of the severity of the condition and often varies between individuals affected by the same genetic mutation and even in the same family (Brook et al. [2]). This is an indication of the complex aetiology of the condition.

The site of the congenitally missing found in previous studies is not random, but relates to position in the dental arch and timing of development [3]. Those teeth that are positioned furthest distally and are the last to develop in their tooth type (incisor/premolar/molar) are those most frequently absent $[4,5]$. This emphasises why, as an integral part of the overall study, in this component we are investigating timing of dental development, which in the complex developmental process may be related to congenital absence as well as smaller size and different crown and root shape.

Recording the distance between specific landmarks is undertaken using the computer programming platform MATLAB which can accept and display an X-ray image in any commonly used format. After highlighting two different landmarks, our program then computes the distance and displays on the screen as well as in the command window. It allows continuous pairwise measurements to avoid restarting the procedure. For anterior teeth, the distance between the Cement Enamel Junction and the root apex is measured [6]. This allows the crown-to-root ratio to be determined; this approach compensates for different tooth angulations between individuals.

For posterior teeth, the method developed by Holt and Brook [7] is used to determine the degree of Taurodontism. The axis between the mesial and distal aspects of the Cement Enamel Junction is taken as the baseline and the distances are measured between this line and the floor of the pulp chamber and the apex of the distal root (Fig. 1). There are conflicting findings in the literature concerning any association between Hypodontia and Taurodontism, possibly related to the failure in some studies to use clear, objective criteria to diagnose Taurodontism.

In addition, we are recording three rooted mandibular first molar teeth, fused molar roots, cuneiform molar roots and invaginations. These additional criteria were also monitored in a study regarding variations of tooth root morphology in a Romano-British population by Brook and Scheers [8].

The MATLAB program has also been adapted to calculate surface area of both the whole tooth as well as the crown and roots separately. These measurements are made by the operator selecting a starting landmark on the radiograph with the mouse and tracing the outline of the 

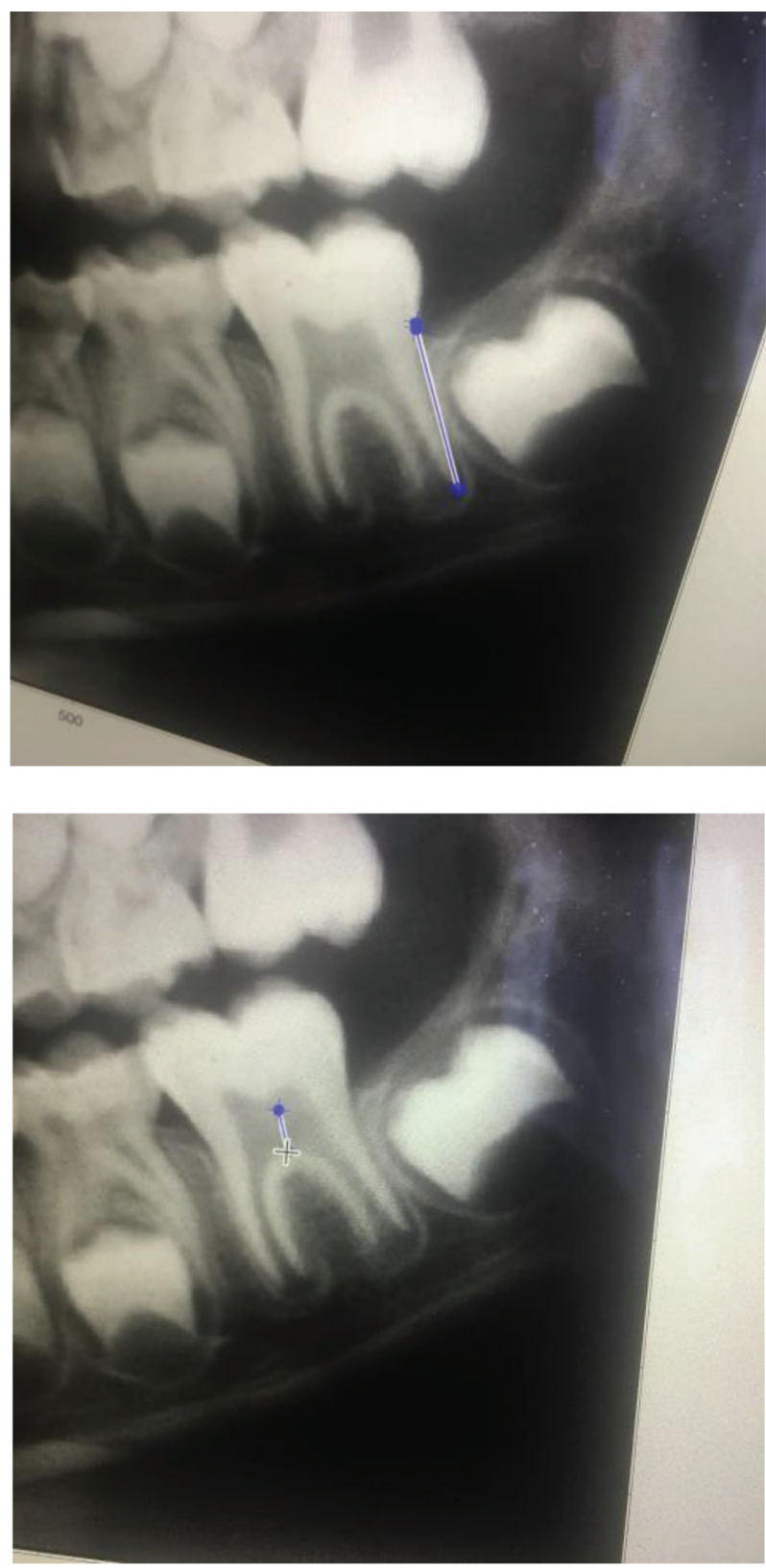

Figure 1: Top: Using the MATLAB system to measure the distance between the Cement Enamel Junction and the apex of the distal root. Bottom: The distance between the Cement Enamel Junction and the floor of the pulp chamber.

area to be measured before clicking again to stop at the end point. The crosshair used to outline the area is seen below in Figure 2 with the extracted area shown in Figure 3.

The program is designed to facilitate the measurement in an automatic way. Since there is possible error margin due to operator using this method, we have built in a curve smoothing 


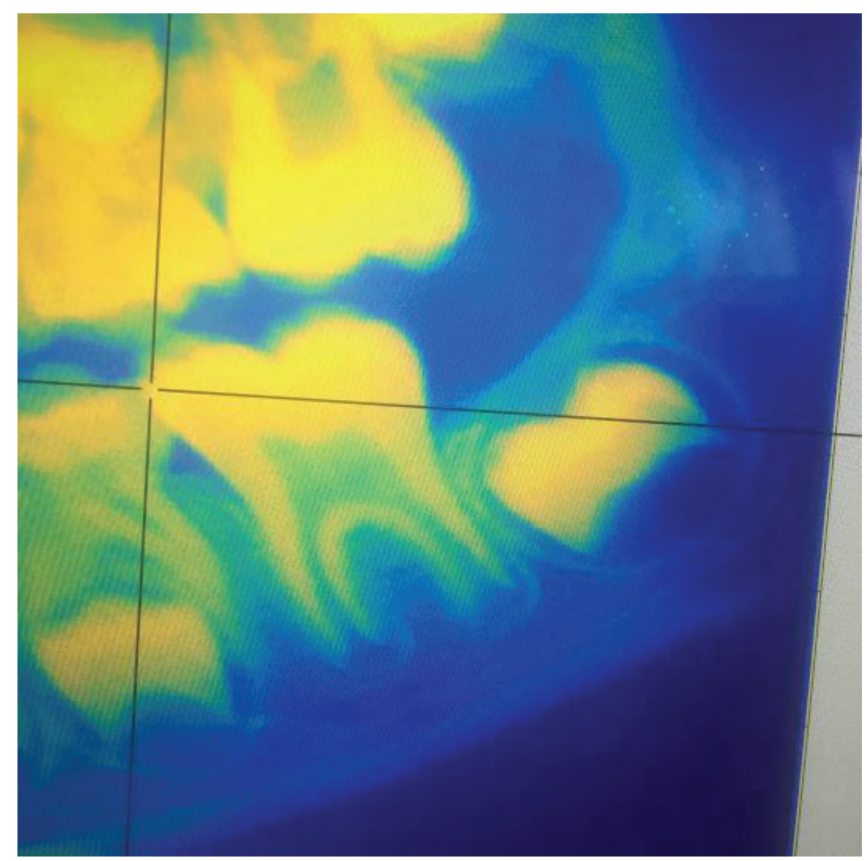

Figure 2: Start of the operator input.

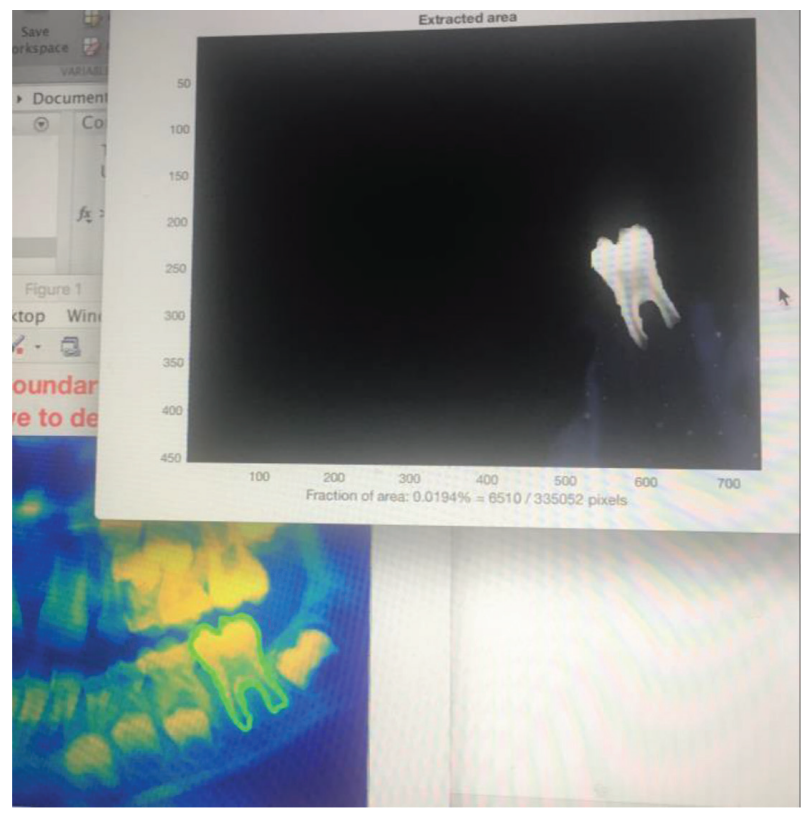

Figure 3: The traced area is then extracted and measured separately by the program. 
function to automatically correct the input so that the final area calculated will be accurate as far as removal of noise or minor hand shaking is concerned. While this correction cannot compensate for major input error, the accuracy of input will be investigated by repeat measurements for intra-operator and inter-operator reproducibility.

This correction procedure can be statistically assessed to inform its reliability using boxplot (Fig. 4). The results from this section allow for the crown-to-root ratio to be measured by a novel approach, which can be compared against compared against existing techniques for accuracy and efficiently.

The program is further being used to measure root angle to gather more information and increase understanding of root morphology. This was perhaps the most challenging part of the study as there was some debate as to the angle we should be measuring. One consideration was
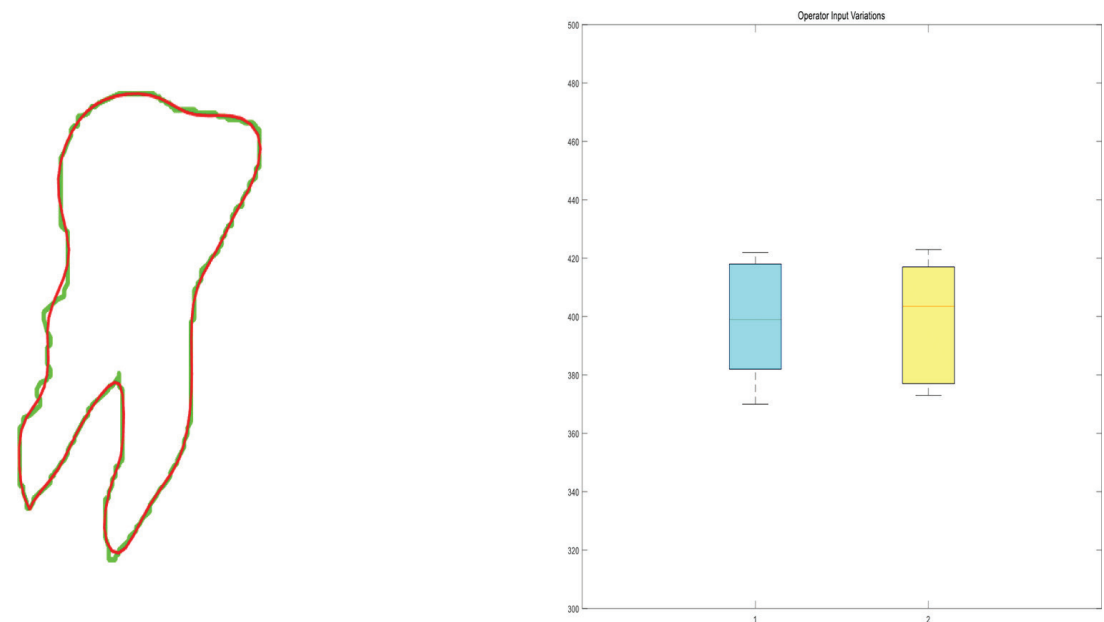

Figure 4: Left: Automatic curve smoothing (red) to reduce input errors (green).Right: Test of input variations (left cyan box for input and right yellow box for corrected values).

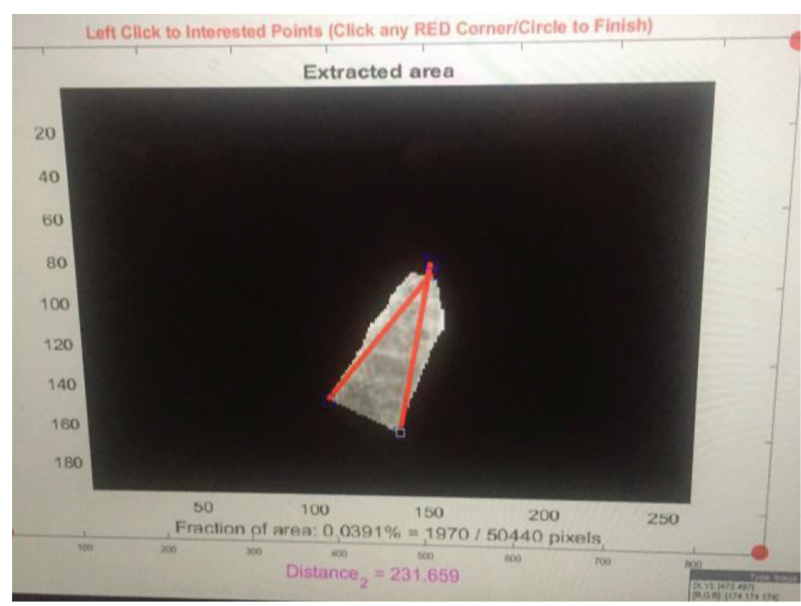

Figure 5: A simple example of operator defined angle. Other automatic ways are also considered. 
to measure the root cone angle which was mentioned by Falk [9]. The method we have developed included selecting the most apical portion of the root and using the angle created by this point and the most coronal mesial and distal aspects of the root. An example of this proposed method is shown as Figure 5 below. We have also considered ways of automatically correcting errors due to operator selection by specifying a region with geometry instead of points and further extracting a more consistent measure of angle information

\section{CONCLUSIONS}

Investigating root morphology and the timing of dental development provides additional insight into the factors in the complex adaptive process that gives rise to hypodontia. This study has advanced the techniques available and provides the basis for the further development of this component of the overall study. The idea of automatically correcting operator input to improve reliability of measurements is of general interest.

\section{REFERENCES}

[1] Brook, A.H. \& O'Donnell, M.B., The dentition: a complex system demonstrating self-* principles, 2011 Fifth International Conference on Self Adaptive and Self Organising Systems. C.P.S. Washington IEEE, pp. 208-209, 2011.

https://doi.org/10.1109/SASO 2011.41

[2] Brook, A., O’Donnell, M.B., Hone, A., Hart, E., Hughes, T., Smith, R. \& Townsend, G., General and craniofacial development are complex adaptive processes influenced by diversity. Australian Dental Journal, 59(S1), pp. 13-22, 2014. https://doi.org/ 10.1111/adj.12158

[3] Townsend, G., Harris, E.F., Lesot, H., Clauss, F. \& Brook, A.H., Morphogenetic fields within the human dentition: a new clinically relevant synthesis of an old concept. Archives of Oral Biology, 54(S1), 34-44, 2009. https://doi.org/10.1016/j.archoralbio.2008.06.011

[4] Brook, A.H., A unifying aetiological explanation for anomalies of human tooth number and size. Archives of Oral Biology, 29, pp. 373-378, 1984. https://doi.org/10.1016/0003-9969(84)90163-8

[5] Kirkham, J., Kaur, R., Stillman, E.C., Blackwell, P.G., Elcock, C. \& Brook A.H., The patterning of hypodontia in a group of young adults in Sheffield. U.K. Archives of Oral Biology, 50, pp. 287-291, 2005. https://doi.org/10.1016/j.archoralbio.2004.11.015

[6] Brook, A.H. \& Holt, R.D., The relationship of crown length to root length in permanent maxillary central incisors. Proceedings of the British Paedodontic Society, 8, pp. 17-20, 1978.

[7] Holt, R.D. \& Brook, A.H., Taurodontism: a criterion for diagnosis and its prevalence in mandibular first permanent molars in a sample of 1,115 British schoolchildren. Journal of the International Association of Dentistry for Children, 10(2), pp. 41-47, 1979.

[8] Brook, A.H. \& Scheers, M., Variation in tooth root morphology in a Romano-British population. Dental Anthropology, 19(2), pp. 33-38, 2006.

[9] Falk, D., Book Review of 'An introduction to human evolutionary anatomy' by L. Aiello and C. Dean. Man, 27, pp. 410-411. https://doi.org/10.2307/2804064 